\title{
Perfil clínico e sociodemográfico de pacientes com Transtorno Afetivo Bipolar em um serviço especializado do Distrito Federal
}

\author{
Clinical and sociodemographic profile of patients with Bipolar Affective Disorder in a \\ specialized service in Distrito Federal
}
Perfil clínico y sociodemográfico de pacientes con trastorno afectivo bipolar en un servicio especializado del Distrito Federal

Beatriz Regis da Cunha ${ }^{1 *}$, Gabriel de Souza², Bráulio Brandão Rodrigues ${ }^{3}$, Lair da Silva Gonçalves ${ }^{3}$.

\section{RESUMO}

Objetivo: Identificar a prevalência e as características dos pacientes bipolares do serviço e relacioná-las com a literatura. Métodos: Realizou-se um estudo descritivo, transversal, quantitativo e com dados coletados dos prontuários de pacientes bipolares de ambulatório terciário no Distrito Federal. Os dados obtidos foram compilados em dois formulários eletrônicos autorais, analisados, descritos em frequências. A pesquisa foi aprovada pelo comitê de ética e pesquisa (CAAE:36765820.6.0000.5553). Resultados: A amostra foi constituída por 84 pacientes, com média de idade de 41,3. Observou-se predomínio de mulheres $(71,4 \%)$, solteiros $(44 \%)$, desempregados $(71,4 \%)$, com ensino médio completo $(39,3 \%)$, sendo $68,7 \%$ diagnosticados como bipolares tipo I. Sobre medicações, os estabilizadores de humor $(90,2 \%)$ e antipsicóticos atípicos $(63,4 \%)$ foram os mais prescritos, sendo que $51,2 \%$ têm histórico de adesão inadequada ao tratamento. Conclusão: Este estudo é relevante, pois se compreende as características, percebe-se falhas nos registros e, com isso, é possível propor medidas de melhorias de atendimento à população.

Palavras-chave: Transtorno bipolar, Prevalência, Epidemiologia.

\section{ABSTRACT}

Objective: To identify the prevalence and characteristics of bipolar patients in the service and relate them to the literature. Methods: A descriptive, cross-sectional, quantitative study was carried out, with data collected from the medical records of bipolar patients at a tertiary outpatient clinic in the Federal District. The data obtained were compiled in two electronic electronic forms, analyzed, described in frequencies. The research was approved by the research ethics committee (CAAE:36765820.6.0000.5553). Results: The sample consisted of 84 patients, with a mean age of 41.3. There was a predominance of women $(71.4 \%)$, single (44\%), unemployed $(71.4 \%)$, with complete high school $(39.3 \%)$, with $68.7 \%$ diagnosed as bipolar I. About medications, mood stabilizers $(90.2 \%)$ and atypical antipsychotics $(63.4 \%)$ were the most prescribed, with $51.2 \%$ having a history of inadequate treatment adherence. Conclusion: This study is relevant, as the characteristics are understood, there are gaps in the records and, with this, it is possible to propose measures to improve the care provided to the population.

Keywords: Bipolar disorder, Prevalence, Epidemiology.

\section{RESUMEN}

Objetivo: Identificar la prevalencia y características de los pacientes bipolares en el servicio y relacionarlas con la literatura. Métodos: Se realizó un estudio descriptivo, transversal y cuantitativo, con datos recolectados de la historia clínica de pacientes bipolares en un ambulatorio terciario del Distrito Federal. Los datos obtenidos fueron recopilados en dos formularios electrónicos electrónicos, analizados, descritos en frecuencias. La investigación fue aprobada por el comité de ética en investigación (CAAE: 36765820.6.0000.5553). Resultados: La muestra estuvo formada por 84 pacientes, con una edad media de 41,3 años. Hubo predominio de mujeres $(71,4 \%)$, solteras $(44 \%)$, desempleadas $(71,4 \%)$, con bachillerato completo $(39,3 \%)$, con un $68,7 \%$ diagnosticado como bipolar I. Sobre medicamentos, estabilizadores del estado de ánimo $(90,2 \%)$ y los antipsicóticos atípicos $(63,4 \%)$ fueron los más prescritos, con un $51,2 \%$ con antecedentes de adherencia inadecuada al tratamiento. Conclusión: Este estudio es relevante, ya que se entienden las características, existen lagunas en los registros y, con ello, es posible proponer medidas para mejorar la atención brindada a la población.

Palabras clave: Trastorno bipolar, Prevalencia, Epidemiología.

\footnotetext{
${ }^{1}$ Universidade de Brasília, Brasília - DF. *E-mail: beatrizrcunha2000@gmail.com

2 Escola Superior de Ciências da Saúde, Brasília - DF.

${ }^{3}$ Hospital São Vicente de Paulo (HSVP) SES-DF, Brasília - DF.
}

Produção incentivada pela Escola Superior de Ciências da Saúde, Brasília-DF. 


\section{INTRODUÇÃO}

O Transtorno Afetivo Bipolar (TAB) é considerado uma patologia psiquiátrica grave e é caracterizada por instabilidade de humor. Ela envolve e afeta questões neuroquímicas, cognitivos, funcionais, familiares e socioeconômicos. O quadro fásico e episódico de depressão e de mania parecido com outros transtornos psiquiátricos torna o diagnóstico complexo e demorado (JÚNIOR PRB, 2019).

Segundo o Manual Diagnóstico e Estatístico de Transtornos Mentais (DSM-5), é classificado em tipo I (um ou mais episódios maníacos ou mistos), tipo II (um ou mais episódios depressivos maiores acompanhados por pelo menos um episódio hipomaníaco), ciclotímicos (perturbação crônica e flutuante do humor) e aqueles sem especificação (AMERICAN PSYCHIATRIC ASSOCIATION, 2014).

Vale ressaltar que o TAB afeta cerca de $1 \%$ a $2 \%$ da população mundial (DEMINCO M, 2018). As manifestações clínicas dos primeiros sintomas ocorrem geralmente na adolescência e em torno de $15 \%$ a $19 \%$ dos indivíduos cometem suicídio (MENEZES SL e SOUZA MC, 2012). Portanto, a patologia em questão prejudica significativamente a qualidade de vida dos pacientes e causa grande sofrimento para suas famílias (MCCORMICK U, et al., 2015).

Conforme a Organização Mundial da Saúde, o transtorno é considerado como a sexta causa de incapacidade e a terceira entre as doenças mentais, ficando depois apenas da depressão unipolar e esquizofrenia (PONT GCD, 2017). A formulação de políticas em saúde mental para a população com este diagnóstico depende essencialmente de informações a respeito das características epidemiológicas, sociodemográficas e clínicas do TAB (LIMA MS, et al., 2005).

Além disso, os pacientes necessitam de terapêuticas que contemplem a multifatorialidade na ocorrência desse transtorno (SANTOS VC, et al., 2017). Várias farmacoterapias são consideradas eficazes e a polifarmácia é frequentemente utilizada, pois alguns agentes podem ser satisfatórios somente em determinadas fases do TAB (MIASSO Al, et al., 2012; JOSHI A, et al., 2019). Dentre as classes de psicotrópicos mais efetivos utilizados no quadro em questão, enquadram-se os anticonvulsivantes, antipsicóticos e o estabilizadores de humor (JOSHI A, et al., 2019).

Dessa forma, o presente estudo mostra-se relevante, pois objetiva executar uma pesquisa observacionaldescritiva, no contexto ambulatorial em um cenário real, para atualizar e compilar informações sobre o perfil da população bipolar da região em questão.

\section{MÉTODOS}

Realizou-se um estudo observacional-descritivo, de caráter transversal e de abordagem quantitativa. Foram analisados 84 prontuários eletrônicos de pacientes com TAB atendidos em um ambulatório especializado de transtorno de humor e ansiedade do Distrito Federal, no período de 2019 a 2021. Importante relatar também que o ambiente é cenário para formação de médicos residentes em psiquiatria. Os critérios de inclusão utilizados foram: pacientes maiores de 18 anos com o diagnóstico de interesse.

Para a coleta dos dados foram elaborados dois formulários eletrônicos, onde as informações provindas dos prontuários eram compiladas. No primeiro, foram incluídos dados sociodemográficos como número de identificação, idade, data de nascimento, sexo, com quem mora, estado de naturalidade, estado conjugal, número de filhos, escolaridade, religião, atividade laboral e história obstétrica das pacientes.

O segundo formulário uniu informações clínicas como diagnóstico/subtipo diagnóstico (TAB tipo 1, TAB tipo 2, suspeita de $T A B, T A B$ ciclotímico, $T A B$ misto), polaridade atual do humor, exames laboratoriais relevantes (2 últimos anos), comorbidades psiquiátricas e clínicas, idade e polaridade do humor no primeiro episódio, número de tentativas de suicídio, histórico de sintomas psicóticos, número de hospitalizações psiquiátricas prévias, uso atual/prévio de substâncias psicoativas, histórico familiar de transtornos psiquiátricos, histórico de baixa adesão terapêutica e os medicamentos utilizados.

Após aplicação dos formulários, os dados foram transferidos para uma base de dados estruturada no formato de planilha, no programa Excel. Posteriormente, foram verificados e corrigidos os erros de digitação 
e, por fim, os dados mais relevantes para a pesquisa foram transportados para serem analisados estatisticamente. As relações entre as variáveis categóricas foram analisadas por meio de estatística descritiva e expressas em frequências absolutas e relativas (\%). A variável contínua (idade) foi expressa em média e desvio padrão.

A pesquisa foi aprovada pelo Comitê de Ética em Pesquisa (CEP), sob o protocolo de número 36765820.6.0000.5553.

\section{RESULTADOS}

A análise dos 84 prontuários demonstra que $71,4 \%(n=60)$ da amostra eram mulheres e 28,6\% ( $n=24)$, homens. A idade média foi de 41,3 \pm 11 anos, sendo a mínima de 23 e a máxima de 69 anos. Quanto à religião: $61,3 \%(n=53)$ sem registro, 20,2\% $(n=17)$ eram evangélicos, $15,5 \%(n=13)$ católicos e 1,2\% $(n=1)$ declararam não ter. Em relação a com quem o paciente vive, 30,5\% $(n=26)$ mora com o cônjuge e com filhos, $19,5 \%(n=16)$ mora com os pais, $13,4 \%(n=11)$ mora sem o cônjuge e com filhos e $8,5 \%$ ( $n=7)$ vive sozinho. Analisando o estado civil, 44\% $(n=37)$ eram solteiros, 36,4\% $(n=31)$ são casados, $10,7 \%(n=9)$ são divorciados e $6 \%(n=5)$ união estável. Considerando a ocupação, 28,6\% possuíam emprego e 71,4\% não possuíam (Tabela 1).

Tabela 1 - Caracterização sociodemográfica dos pacientes bipolares atendidos no serviço em questão.

\begin{tabular}{lcc}
\hline Variável & $\mathbf{N}$ & $\%$ \\
\hline Laboral ativo & & \\
\hline Autônomo & 8 & 3,5 \\
Motorista & 3 & 3,6 \\
Não se sabe & 60 & 3,4 \\
Professor & 3 & 9,6 \\
Serviços gerais & 8 & 1,2 \\
Servidor Público & 1 & 1,2 \\
Vendedor & 1 & \\
\hline Laboral inativo & & 10,7 \\
\hline Aposentado & 9 & 3,6 \\
Auxílio-Doença & 3 & 3,6 \\
Benefício de Prestação Continuada & 3 & 51,2 \\
Desempregado & 43 & 28,6 \\
Não se sabe & 24 & 2,4 \\
Outro & 2 & \\
\hline Escolaridade & & 7,1 \\
\hline Ensino Fundamental I incompleto & 6 & 15,6 \\
Ensino Fundamental I completo & 3 & 2,4 \\
Ensino Fundamental II incompleto & 13 & 4,8 \\
Ensino Fundamental II completo & 2 & 39,3 \\
Ensino Médio incompleto & 4 & 8,3 \\
Ensino Médio completo & 33 & 8,3 \\
Graduação incompleta & 7 & 10,7 \\
Graduação completa & 7 & \\
Não se sabe & 9 & \\
\hline Fon & & \\
\hline
\end{tabular}

Fonte: Cunha BR, et al., 2021.

Além disso, 26,8\% $(n=15)$ das pacientes tiveram nenhuma gestação, $25 \%(n=14)$ tiveram uma única, outras $26,8 \%(n=15)$ tiveram duas gestações e $21,5 \%(n=12), 3$ ou mais. Encontrou-se taxa de laqueadura indicada em 13,3\% ( $n=8)$ e uso de algum contraceptivo em 18,3\% $(n=11)$. Por fim, 20,3\% $(n=12)$ tiveram depressão pós-parto e 39\% $(n=23)$ não tiveram. Também foram coletados os dados referentes às comorbidades clínicas apresentadas pelos pacientes (Tabela 2). 
Tabela 2 - Dados clínicos dos pacientes bipolares atendidos no serviço em questão.

\begin{tabular}{lcc}
\hline Variável & N & $\%$ \\
\hline Comorbidades clínicas & & \\
\hline Sim & 35 & 41,7 \\
Não & 49 & 58,3 \\
\hline Se sim: & 1 & \\
\hline Chagas & 1 & 1,2 \\
Dislipidemia & 3 & 1,2 \\
Diabetes Mellitus (DM) & 1 & 3,6 \\
Doença do Refluxo Gastroesofágico & 1 & 1,2 \\
Enxaqueca & 1 & 1,2 \\
Epilepsia & 5 & 1,2 \\
Hipertensão Arterial (HAS) & 2 & 6,0 \\
HAS e DM & 1 & 2,4 \\
HAS, DM e dislipidemia & 11 & 1,2 \\
Hipotireoidismo & 1 & 13,1 \\
HIV e DM & 6 & 1,2 \\
Obesidade & 1 & 7,2 \\
Obesidade e DM & & 1,2 \\
\hline
\end{tabular}

Fonte: Cunha BR, et al., 2021.

Na primeira crise, 67,9\% ( $n=57)$ dos pacientes tiveram episódio maníaco, 26,2\% ( $n=22)$ tiveram episódio depressivo e $6 \%(n=5)$, misto. Tratando-se de subtipo diagnóstico, $68,7 \%$ dos casos $(n=58)$ foram classificados clinicamente como Tipo I, [24,1\% ( $n=20)]$ Tipo II, [6\% ( $n=5)]$ como casos suspeitos e $1,2 \%(n=1)$ com TAB Misto. Também foram encontrados dados relativos à suicidalidade dos pesquisados e sobre 0 número de internações prévias (Tabela 3).

Tabela 3 - Dados da história psiquiátrica dos pacientes bipolares atendidos no serviço em questão.

\begin{tabular}{lcc}
\hline Variável & N & $\%$ \\
\hline Idade da primeira crise & & \\
\hline Entre 15 e 25 anos & 35 & 41,7 \\
Mais de 25 anos & 40 & 47,6 \\
Mais de 45 anos & 5 & 6,0 \\
Menos de 15 anos & 4 & 4,8 \\
\hline Tentativas de suicídio & & \\
\hline Nenhuma & 53 & 63,1 \\
Uma & 20 & 23,8 \\
Duas & 5 & 6,0 \\
Três & 3 & 3,6 \\
Diversas vezes & 3 & 3,6 \\
\hline Se sim: & & \\
\hline "tentou se jogar na frente dos carros" & 4 & 4,8 \\
Arma branca & 4 & 4,8 \\
Arma de fogo & 1 & 1,2 \\
Afogamento & 1 & 1,2 \\
Enforcamento & 5 & 6,0 \\
Ingestão de medicamentos & 12 & 14,3 \\
Não identificado & 4 & 4,8 \\
\hline Internações & & \\
\hline 0,00 & 34 & 40,5 \\
1,00 & 27 & 32,1 \\
2,00 & 9 & 10,7 \\
3,00 & 9 & 10,7 \\
4,00 & 5 & 6,0 \\
\hline
\end{tabular}

Fonte: Cunha BR, et al., 2021. 
Sobre os exames laboratoriais encontrados nos prontuários, evidenciou-se uma grande parcela de dados não informados ou não registrados. As informações mais relevantes encontradas foram as de que alguns pacientes tinham parâmetros acima da referência, tais como $17.9 \%(n=15)$ para TGO (transaminase oxalacética), 25\% ( $n=21$ ) para TGP (transaminase pirúvica), 22.6\% $(n=19)$ para glicemia de jejum; 7.3\% $(n=6)$ para hemoglobina glicada, $13.1 \%(n=11)$ para TSH (hormônio tireoestimulante), $20.2 \% \quad(n=17)$ para triglicerídeos e $16.9 \%(n=14)$ para colesterol total. Por fim, para a litemia entre pacientes que encontravamse em uso de lítio, encontrou-se que 15.7\% ( $n=13)$ tinham níveis menores que 0,6 e 7.2\% (n=6) acima de 0,9.

Quanto ao histórico familiar de transtornos mentais, 66,3\% ( $n=55)$ dos pacientes analisados apresentavam alguma doença mental associada na família. Também foram analisados o uso atual e/ou prévio de substâncias psicoativas (Tabela 4).

Tabela 4 - Uso atual ou prévio de substâncias psicoativas pelos pacientes bipolares atendidos no serviço em questão.

\begin{tabular}{lcc}
\hline Variável & $\mathbf{N}$ & $\%$ \\
\hline Tabagismo & & \\
\hline 10 cigarros/dia & 1 & 1,2 \\
5 cigarros/dia & 1 & 1,2 \\
2 cigarros/dia & 3 & 3,6 \\
Ex-tabagista & 8 & 9,5 \\
Não & 71 & 84,5 \\
\hline Etilismo & & \\
\hline Etilismo crônico & 3 & 3,6 \\
Não & 73 & 86,7 \\
Ocasional & 8 & 9,5 \\
\hline Outros & & \\
\hline Cocaína & 1 & 1,2 \\
Energético & 1 & 1,2 \\
Maconha & 5 & 6,0 \\
Não & 77 & 91,7 \\
\hline
\end{tabular}

Fonte: Cunha BR, et al., 2021.

Considerando o histórico de adesão terapêutica, identificou-se que 51.2\% ( $n=43)$ tiveram índices considerados baixos. Por fim, em se tratando dos medicamentos usados, foram identificados estabilizadores de humor (90,2\%) antipsicóticos típicos (46,3\%) e atípicos (63,4\%), antidepressivos (15,9\%), ansiolíticos benzodiazepínicos (15,9\%), não benzodiazepínicos (2,4\%) e antiparkinsonianos (18,3\%) (Tabela 5).

Tabela 5 - Medicamentos usados pelos pacientes bipolares atendidos no serviço em questão.

\begin{tabular}{lcc}
\hline Variável & N & $\%$ \\
\hline Estabilizadores de humor & & \\
\hline Carbonato de lítio & 40 & 52,6 \\
Carbonato de lítio ER & 1 & 1,3 \\
Divalproato ER & 3 & 3,9 \\
Ácido Valpróico & 50 & 65,8 \\
Lamotrigina & 6 & 7,9 \\
Carbamazepina & 1 & 1,3 \\
Topiramato & 4 & 5,3 \\
\hline Antipsicóticos típicos & & \\
\hline Haloperidol & 2 & 5 \\
Clorpromazina & 11 & 27,5 \\
Levomepromazina & 15 & 37,5 \\
Tioridazina & 0 & 0 \\
Haloperidol decanoato & 9 & 22,5 \\
Zuclopentixol depot & 8 & 20 \\
\hline
\end{tabular}




\begin{tabular}{lcc}
\hline Variável & N & $\%$ \\
\hline Antipsicóticos atípicos & 14 & 26,4 \\
\hline Risperidona & 14 & 26,4 \\
Quetiapina & 4 & 7,5 \\
Aripiprazol & 1 & 1,9 \\
Ziprasidona & 3 & 5,7 \\
Clozapina & 0 & 0 \\
Lurasidona & 19 & 35,8 \\
Olanzapina & & \\
\hline Antidepressivos & 4 & 33,3 \\
\hline Fluoxetina & 1 & 8,3 \\
Sertralina & 2 & 16,7 \\
Paroxetina & 1 & 8,3 \\
Citalopram & 2 & 16,7 \\
Bupropiona & 3 & 25 \\
Amitriptilina & & \\
\hline Ansiolíticos/ Hipnóticos Benzodiazepínicos & 11 & 78,6 \\
\hline Clonazepam & 4 & 28,6 \\
Diazepam & 0 & 0 \\
Alprazolam & 1 & 7,1 \\
Lorazepam & & \\
\hline Antiparkinsonianos & 7 & 43,8 \\
\hline Prometazina & 76,3 \\
Biperideno & 9 & 6,3 \\
Amantadina & 1 &
\end{tabular}

Fonte: Cunha BR, et al., 2021.

\section{DISCUSSÃO}

\section{Sexo}

Um aspecto interessante observado durante a pesquisa é o fato das mulheres formarem $71,4 \%$ dos pacientes diagnosticados com TAB, contrariando estudos que apontam que não há prevalência de sexo entre os indivíduos (JÚNIOR PRB, 2019). Entretanto, tal dado pode ser explicado pelo fato dos homens procurarem significativamente menos atendimento médico, seja por resistência pessoal, seja por estigma social (RÉUS $\mathrm{PH}$, et al., 2019).

Vale ressaltar que o manejo clínico da doença bipolar nas mulheres apresenta particularidades importantes que dificultam o tratamento, como as variações hormonais no ciclo reprodutivo, em especial na gestação onde há aumento do risco de recorrência do TAB, especialmente se os medicamentos forem interrompidos e que dificultam a escolha destes na tentativa de evitar riscos de teratogenicidade para 0 feto e 0 neonato (MCCORMICK U, et al., 2015; MIASSO Al, et al., 2012). Aliado a isso, verifica-se que somente cerca de $20 \%$ dos prontuários das pacientes especificaram sua história gestacional e apenas $18,3 \%$ incluíram o uso de métodos contraceptivos, evidenciando uma carência de informações registradas nos documentos hospitalares em questão.

\section{Idade}

O TAB tem diversas outras patologias que também transitam entre mania, hipomania e depressão como diagnóstico diferencial. Devido a essa proximidade, os pacientes costumam ser diagnosticados tardiamente, entre os 20 e 30 anos e os dados encontrados confirmam tal constatação (JÚNIOR PRB, 2019; RÉUS PH, et al., 2019). Dessa forma, um diagnóstico precoce possibilitaria um melhor suporte e terapêutica, já que o início do quadro clínico é um momento crucial para se estabelecer uma aliança de qualidade com o paciente e facilitar a introdução e adesão terapêutica (MIASSO Al, et al., 2012; MCCORMICK U, et al., 2015; ENES CL, et al., 2020). 


\section{Estado civil}

Da mesma forma como traz Júnior PRB (2019), os resultados apontam índices elevados de divórcio $(10,7 \%)$ nesses pacientes, além de uma expressiva parte serem solteiros (44\%). Atribui-se esses índices a crises relacionadas ao transtorno, com turbulência e incapacidade de manter relações saudáveis a longo prazo (JÚNIOR PRB, 2019). Além disso, a taxa de divórcios desses pacientes é aproximadamente de duas a três vezes maior, quando comparada à de indivíduos não portadores da doença (MIASSO AI, et al., 2012). O percentual mais elevado de indivíduos sem parceiro(a) pode justificar-se também pelas limitações desencadeadas pela doença, como disfunção sexual (pelo uso de fármacos), oscilações do humor e dificuldades no cuidado dos filhos (ENES CL, et al., 2020).

Vale destacar que a maioria dos pacientes deste estudo expressa o TAB tipo 1 (68,7\%) que caracteriza episódios que oscilam entre depressão e mania, evidenciando a inconstância clínica e podendo refletir diretamente no modo como se dão os relacionamentos interpessoais (JÚNIOR PRB, 2019; LOPES FL, 2012).

\section{Ocupação}

Constatou-se um expressivo número de pacientes desempregados $(71,4 \%)$, o que corrobora com a literatura. Existem fortes indícios de que os efeitos colaterais dos medicamentos e as experiências de episódios de crise durante o trabalho impactam significativamente na manutenção dos empregos (JúNIOR PRB, 2019; MCCORMICK U, et al., 2015). Eles podem apresentar dificuldades mesmo após remissão da sintomatologia, devido aos efeitos residuais da doença bem como com o estigma do transtorno psiquiátrico (LOPES FL, 2012).

Conforme Sadana D, et al. (2019) e Weintraub MJ, et al. (2021), pacientes em fase eutímica tiveram pontuações baixas a respeito da velocidade de processamento de informações em comparação com controles saudáveis. E, segundo VARO C, et al. (2019), pacientes bipolares obtiveram escores mais baixos em comparação com os controles saudáveis no Quociente de Inteligência Emocional e no ramo Compreendendo as Emoções. Outros fatores como insônia, depressão e alguns medicamentos podem perturbar a atenção podem causar lentidão motora (KATO T, 2019), influenciando diretamente na ocupação destes indivíduos.

\section{Escolaridade}

A maioria dos pacientes $(55,9 \%)$ completaram o ensino médio. Esse resultado pode ser explicado pelo fato do ambulatório em questão estar localizado em Brasília, onde se tem melhor qualidade de vida. Isto sugere um elevado índice de instrução dos pacientes e, consequentemente, uma melhor compreensão da sua doença e adesão ao tratamento. Contudo, este dado vai contra os encontrados em estudos prévios na literatura, revelando que apesar do TAB causar manifestações de sintomas que limitam a atividade laboral, social e de lazer, neste estudo não desencadeou uma menor taxa de escolarização (SCUSSEL F, et al., 2016).

\section{Subtipos e especificadores}

A classificação TAB tipo I foi a mais encontrada, com $68,7 \%$ dos pacientes. Tal prevalência também é considerada como maior na literatura em geral quando se trata deste subtipo (RÉUS PH, 2019). Apesar disso, é importante considerar como limitação o fato dos indivíduos terem sido diagnosticados por critérios clínicos e não por meio de entrevistas estruturadas, como a escala SCID.

Não foi possível obter dados tabulados quanto aos especificadores tendo em vista a falta de registros padronizados nos prontuários eletrônicos e não terem sido encontrados dados de escalas que avaliam humor, tais como Young ou Hamilton. Porém, é sabido que a presença de características psicóticas (encontradas na história de em $85,7 \%$ dos pacientes) está associada à maior proporção de tempo em episódios de doença e à maior taxa de recorrência (TONDO L, et al., 2017).

\section{Histórico familiar}

Há uma forte associação genética em torno desta patologia e seu caráter hereditário (KATO T, 2019). Assim, 66,3\% $(n=55)$ dos pacientes analisados apresentavam algum transtorno mental na família, número 
acima dos encontrados na literatura brasileira (em torno de 20\% para histórico positivo de TAB em familiares de 1ํㅡㅁau) (LOPES FL, 2012). Tal resultado justifica-se pelo fato de que foi incluído no questionário qualquer histórico familiar de doença psiquiátrica e não somente o diagnóstico do TAB.

\section{Farmacologia}

As medicações identificadas encontram-se entre as indicadas na literatura para o transtorno. De acordo com as diretrizes do National Institute for Health and Care Excellence (NICE) (2018), os estabilizadores do humor são indicados como tratamento para crises agudas e de manutenção, porém estão associados a efeitos colaterais graves, embora raros. O ácido valpróico foi o mais prescrito $(65,8 \%)$ e é uma das principais escolhas psicofarmacológicas no quadro, porém pode gerar efeitos colaterais como queda de cabelo e alterações nas enzimas hepáticas. O carbonato de lítio, encontrado em $52,6 \%$ pode estar relacionado à redução de taxas de suicídio em alguns estudos e ter baixo custo, facilitando, portanto, o acesso de populações carentes ao medicamento (LOPES FL, 2012; JÚNIOR PRB, 2019; NICE, 2018; JOSHI A, et al., 2019).

Os antipsicóticos atípicos, utilizados nos quadros maníacos agudos (seja em manutenção ou associados aos estabilizadores do humor) demonstram eficácia e segurança aceitável (NICE, 2019). Porém, apenas a quetiapina (26,4\% dos pesquisados) têm eficácia comprovada como monoterapia para a abordagem de episódios depressivos agudos de TAB. Dentre os encontrados, estão o aripiprazol (7,5\%), olanzapina (35,8\%), quetiapina e a ziprasidona (1,9\%) (MCCORMICK U, et al., 2015).

Em relação aos antipsicóticos típicos, demonstrou-se que a levomepromazina foi identificada em 37,5\% dos pacientes, clorpromazina em $27,5 \%$ e o haloperidol em 22,5\%. De forma geral, a classe não teve destaque na literatura pelo fato de que a terapia medicamentosa de primeira escolha, na maioria dos casos, são os antipsicóticos atípicos combinados com estabilizadores do humor e também pelo fato de que podem gerar mais efeitos extrapiramidais quando comparados com os atípicos (JOSHI A, et al., 2019).

Em geral, o uso adequado de antidepressivos convencionais é uma área de controvérsia no tratamento deste transtorno (PACCHIAROTTI I, et al., 2013). A principal preocupação é o seu uso como monoterapia e o risco de precipitar uma mudança para mania/hipomania, que é estimada para ocorrer entre $3 \%$ e $15 \%$ dos casos (MCCORMICK U, et al., 2015). Assim, diretrizes de tratamento recomendam o uso de antidepressivos apenas como um complemento aos estabilizadores de humor para pacientes com transtorno bipolar em fase depressiva (HIRSCHFELD RM, 2014; MCCORMICK U, et al., 2015).

A necessidade de sono reduzida costuma ser uma característica do TAB principalmente no que tange a fase maníaca. O uso de benzodiazepínicos frequentemente é receitado e os achados não foram diferentes, sendo a classe farmacológica presente em 16 pacientes (JÚNIOR PRB, 2019).

\section{Adesão medicamentosa}

Bipolares apresentam tendência à cronicidade e a adequada adesão medicamentosa é imprescindível para possibilitar a melhora do prognóstico. No tratamento, a monoterapia é exceção, o que é considerado como uma das principais dificuldades para a adesão do paciente à terapêutica: a prescrição de vários medicamentos simultaneamente pode resultar em efeitos indesejados e interações medicamentosas (MIASSO Al, et al., 2012).

Outras razões para a baixa adesão entre os pacientes incluem a negação do diagnóstico, a falta de crença de que os medicamentos oferecidos são necessários ou eficazes, o acesso precário aos cuidados de saúde e recursos limitados para apoiar os custos do tratamento ou até mesmo os sintomas de melhora iniciais (SANTOS VC, et al., 2017; JOSHI A, et al., 2019; MCCORMICK U, et al., 2015).

\section{Uso/abuso de substâncias psicoativas}

O uso de substâncias psicoativas (álcool, cigarro e outras drogas) é comorbidade psiquiátrica frequente nos bipolares. O abuso de álcool nesses pacientes está associado a um risco elevado de suicídio, particularmente na presença de transtornos por uso de drogas concomitantes. Um estudo investigou essa associação e concluiu que as taxas mais altas de tentativas de suicídio em pacientes com TAB tipo I e alcoolismo foram explicadas principalmente por pontuações mais altas de agressão (MCCORMICK U, et al., 2015). 


\section{Suicídio}

Foi observado que 36,9\% ( $n=32$ ) dos pacientes do estudo já tentaram o suicídio. Segundo Mccormick $U$, et al. (2015), as taxas de suicídio no TAB são as mais altas entre os transtornos psiquiátricos. A incidência ao longo da vida de pelo menos uma tentativa de suicídio foi relatada nesse estudo como sendo de $29 \%$ em pacientes com TAB, em comparação com 16\% para Transtorno Depressivo Maior.

A bipolaridade é caracterizada por uma instabilidade de humor e associada à impulsividade, abuso de drogas e comportamento sexual inadequado. Além disso, muitos pacientes procuram atendimento por apresentarem sintomas depressivos e são diagnosticados equivocadamente como depressivos unipolares. Isso pode ser possível, pois alguns pacientes manifestam tardiamente quadros maníacos ou hipomaníacos. Esse engano pode induzir novos episódios maníacos e então desestabilizar a doença (HIRSCHFELD RM, 2014; MCCORMICK U, et al., 2015). Assim, devido a estes problemas citados, indivíduos bipolares apresentam mortalidade elevada por suicídio.

Ademais, a grande maioria das tentativas foi realizada através de intoxicação medicamentosa $(40,6 \%)$, 0 que possivelmente se justifica pelo acesso a este meio uma vez que grande parte desses pacientes utilizam medicações que podem levar a óbito, se utilizadas de forma inadequada (LOPES FL, 2012).

\section{Comorbidades clínicas}

O TAB sabidamente vem acompanhado de comorbidades, muitas delas associadas a medicações que podem promover aumento de peso, dislipidemia e aumentam as chances de eventos cardiovasculares. Na análise em questão, 41,7\% ( $n=35)$ dos pacientes apresentaram algum tipo de comorbidade clínica, entretanto não foi possível fazer associações entre elas, já que o desenho de estudo transversal não permite em geral identificar a temporalidade dos fatos (JÚNIOR PRB, 2019). Também foram identificados $13,1 \%(n=11)$ de pacientes com hipotireoidismo, doença comum no sexo feminino e possível efeito colateral do uso do lítio, ambos muito presentes na amostra analisada (LOPES FL, 2012).

\section{Internações}

Foi constatado na amostra que 59,5\% ( $n=50)$ pacientes já tiveram internações psiquiátricas, o que pode indicar maior gravidade dos episódios e pior prognóstico da doença.

\section{Exames laboratoriais}

A respeito dos exames laboratoriais, muitos deles não tiveram documentação nos prontuários nos últimos 2 anos, principalmente a hemoglobina glicada (76.8\%), litemia $(67,5 \%)$ e colesterol total $(49,4 \%)$. Assim, caberia novo estudo para compreender se essa subnotificação se deve à dificuldade de acesso, restrições do SUS no contexto da pandemia por covid-19, à baixa adesão ou baixa renda do paciente.

\section{CONCLUSÃO}

Portanto, os resultados deste estudo foram condizentes com grande parte da literatura apresentada e corroboraram com a informação de que o Transtorno Afetivo Bipolar tem grande impacto na qualidade de vida dos indivíduos, ao afetar a saúde física e mental, além de contribuir para prejuízos na vida social, laboral e pessoal. Como limitações, observou-se que muitas informações não foram adequadamente registradas no prontuário eletrônico e a formulação do diagnóstico deveu-se à história e observação clínica, não tendo sido utilizados entrevistas e escalas adequadas. Dessa forma, a pesquisa contribui para o conhecimento das características da população em questão e propõe o aprimoramento do diagnóstico, dos registros médicos, na adesão medicamentosa e em novos estudos na área.

\section{AGRADECIMENTOS E FINANCIAMENTO}

Agradecimentos aos profissionais que pertencem ao ambulatório em questão por participaram direta ou indiretamente da pesquisa, contribuindo para que os dados fossem colhidos com tranquilidade e de maneira satisfatória. O estudo teve incentivo financeiro por parte da Escola de Ciências da Saúde-DF e compôs o Projeto de Iniciação Científica dos estudantes de medicina. 


\section{REFERÊNCIAS}

1. AMERICAN PSYCHIATRIC ASSOCIATION. Manual Diagnóstico e Estatístico de Transtornos Mentais (DSM-5). 5 ed. Porto Alegre: Artmed, 2014.

2. DEMINCO M. Transtorno Bipolar - Aspectos Gerais. Revista Eletrônica do Psicologia.pt, 2018.

3. ENES CL, et al. Predição da adesão ao tratamento e qualidade de vida de pacientes com transtorno bipolar. Revista de Enfermagem do Centro Oeste Mineiro, 2020; 10: e3489.

4. HIRSCHFELD RM. Differential diagnosis of bipolar disorder and major depressive disorder. Journal Affect Disord, 2014; 169(Suppl 1):S12-6.

5. JOSHI A, et al. Pharmacological Therapies in Bipolar Disorder: a Review of Current Treatment Options. Psychiatria Danubia, 2019; 31(Suppl 3):595-603.

6. JÚNIOR PRB. Perfil epidemiológico dos pacientes com Transtorno Afetivo Bipolar em serviço especializado. Trabalho de Conclusão de Curso (Graduação em Medicina) Universidade Federal da Fronteira Sul, 2019.

7. KATO T. Current understanding of bipolar disorder: Toward integration of biological basis and treatment strategies. Psychiatry and Clinical Neurosciences, 2019; 73(9):526-540.

8. LIMA MS, et al. Epidemiologia do Transtorno Bipolar. Archives of Clinical Psychiatry, 2005. 32 (suppl 1).

9. LOPES FL. Transtorno Bipolar do Humor na atualidade: resultados preliminares de um centro especializado em transtornos afetivos. Monografia (Graduação em Medicina) - Faculdade de Medicina da Bahia. Universidade Federal da Bahia, 2012.

10. MCCORMICK U, et al. Diagnosis and treatment of patients with bipolar disorder: A review for advanced practice nurses. Journal of the American Association of Nurse Practitioners, 2015; 27(9): 530-42.

11. MENEZES SL, SOUZA MC. Implicações de um grupo de Psicoeducação no cotidiano de portadores de Transtorno Afetivo Bipolar. Revista da Escola de Enfermagem da USP, 2012; 46(1).

12. MIASSO AI, et al. Transtorno afetivo bipolar: perfil farmacoterapêutico e adesão ao medicamento. Revista da Escola de Enfermagem da USP, 2012; 46(3).

13. NATIONAL COLLABORATING CENTRE FOR MENTAL HEALTH. The NICE Guideline on the Assessment and Management of Bipolar Disorder in Adults, Children and Young People in Primary and Secondary Care. 2018. Disponível em: http://www.ncbi.nlm.nih.gov/books/NBK498655. Acessado em: 15 de julho de 2021.

14. NATIONAL INSTITUTE OF CLINICAL EXCELLENCE (NICE). Bipolar Disorder: Assessment and Management. 2019. Disponível em: https://www.nice.org.uk/guidance/cg185. Acessado em: 17 de julho de 2021.

15. PACCHIAROTTI I, et al. The International Society for Bipolar Disorders (ISBD) task force report on antidepressant use in bipolar disorders. American Journal of Psychiatry, 2013; 170(11): 1249-62.

16. PONT GCD. Lítio e Valproato agem sobre a via de sinalização de Pi3k/akt/gsk-3ß revertendo o comportamento do tipo maníaco de ratos submetidos a um modelo animal de mania induzido pela Ouabaína. Dissertação (Mestrado em Ciências da Saúde) - Programa de Pós-Graduação em Ciências da Saúde. Universidade do Extremo Sul Catarinense, 2017.

17. RÉUS PH, et al. Perfil Epidemiológico dos Pacientes Bipolares das Clínicas Integradas da Universidade do Extremo Sul Catarinense. Revista Brasileira de Psiquiatria, 2019.

18. SADANA D, et al. Neurocognitive profile of patients with Bipolar Affective Disorder in the euthymic phase. Asian Journal of Psychiatry, 2019; 44: 121-126.

19. SANTOS VC, et al. Transtorno Afetivo Bipolar: Terapêuticas, Adesão ao Tratamento e Assistência de Enfermagem. Revista Brasileira De Saúde Funcional, 2017; 1(1): 10.

20. SCUSSEL F, et al. Perfil Clínico dos Pacientes com Transtorno Bipolar Atendidos em um Ambulatório Especializado na Região Sul Catarinense. Arquivos Catarinenses De Medicina, 2016; 45(4): 3-10.

21. TONDO L, et al. Depression and Mania in Bipolar Disorder. Current Neuropharmacology, 2017; 15(3): $353-358$.

22. VARO C, et al. Social cognition in bipolar disorder: the role of sociodemographic, clinical, and neurocognitive variables in emotional intelligence. Acta Psychiatrica Scandinavica, 2019; 139(4): 369-380.

23. WEINTRAUB MJ, et al. Social impairment in relation to clinical symptoms in youth at high risk for bipolar disorder. Early Intervention in Psychiatry, 2021. 